\title{
Spinal Cord Infarction in a Patient Undergoing Veno-arterial Extracorporeal Membrane Oxygenation
}

\author{
Beomsu Shin ${ }^{1,2}$, Yang Hyun Cho ${ }^{3}$, Jin-Ho Choi ${ }^{4}$, Jeong Hoon Yang ${ }^{1,4}$ \\ ${ }^{1}$ Department of Critical Care Medicine, ${ }^{2}$ Division of Pulmonary and Critical Care Medicine, Department of Medicine, ${ }^{3}$ Department of Thoracic and \\ Cardiovascular Surgery, and ${ }^{4}$ Division of Cardiology, Department of Medicine, Samsung Medical Center, Sungkyunkwan University School of Medicine, \\ Seoul, Korea
}

Spinal cord infarction is an uncommon, but serious disorder characterized by severe motor impairment and bladder and bowel dysfunction. Spinal cord infarction is likely caused by hypoperfusion at the thoraco-lumbar spinal cord due to diverse reasons. An 81-year-old woman without motor or neurologic dysfunction presented with cardiogenic shock due to acute myocardial infarction. We performed veno-arterial extracorporeal membrane oxygenation (VA ECMO) to maintain adequate organ perfusion. Lower limb weakness was noted on day 1 of ECMO support. Although the symptom persisted, we could not carry out further evaluation because of her hemodynamic instability. After removal of ECMO, spinal magnetic resonance imaging was performed and showed a signal abnormality extending from the level of T5 to the conus medullaris. The patient underwent conservative management, but eventually experienced limb paralysis. Herein, we report a case of spinal cord infarction in a patient with myocardial infarction during VA ECMO support.

Key Words: extracorporeal membrane oxygenation; spinal cord infarction

Spinal cord infarction may be the cause of hemodynamic issues such as embolism, thrombus, or hypotension [1]. The condition is clinically characterized by sudden sensory and motor loss below the level of the spinal cord injury [2]. In case of thoraco-abdominal surgery, and in absence of protective therapies, paralysis after elective repair of degenerative aneurysms has been reported to occur in $5 \%$ of patients [3]. Early and differential diagnosis is important because of the risk of severe neurological damage and complications [4]. Extracorporeal membrane oxygenation (ECMO) is an extracorporeal technique that temporarily supports tissue oxygenation in patients with life-threatening cardiac failure. Bleeding, vessel perforation, and distal ischemia are relatively common complications, but spinal cord infarction is of very rare occurrence in patients undergoing ECMO $[5,6]$. Herein, we present a case of a spinal cord infarction in a patient undergoing ECMO.

\section{CASE REPORT}

An 81-year-old female patient $(150 \mathrm{~cm}, 55.8 \mathrm{~kg}$, American Society of Anesthesiologists physical status grade II) with a history of hypertension and diabetes mellitus presented cardiac arrest requiring mechanical circulatory support. Before she was transported to our hospital,

\section{Case Report}

Received: June 14, 2016

Revised: September 7, 2016

Accepted: September 13, 2016

Corresponding author

Jeong Hoon Yang

Department of Critical Care

Medicine, Samsung Medical Center,

Sungkyunkwan University School of

Medicine, 81 Irwon-ro, Gangnam-gu,

Seoul 06351, Korea

Tel: +82-2-3410-1096

Fax: +82-2-2148-7088

E-mail: jhysmc@gmail.com

Copyright () 2018 The Korean Society of Critical Care Medicine

This is an Open Access article distributed under the terms of Creative Attributions Non-Commercial License (http:// creativecommons.org/li-censes/by-nc/4.0/) which permits unrestricted noncommercial use, distribution, and reproduction in any medium, provided the original work is properly cited. 
bystander cardiopulmonary resuscitation was done for $1 \mathrm{~min}$ ute. At the time of arrival to the emergency room, the Glasgow Coma Scale score was 4 , the initial vital signs included blood pressure as $61 / 35 \mathrm{mmHg}$, heart rate as $56 / \mathrm{min}$, and her room air arterial blood gas levels showed $\mathrm{pH}$ of $7.38, \mathrm{PaO}_{2}$ as 87.6 $\mathrm{mmHg}, \mathrm{PaCO}_{2}$ as $21.1 \mathrm{mmHg}$, and saturation as $96 \%$. Electrocardiogram showed ST-segment elevation on the II, III, and aVF leads with reciprocal changes on the I and aVL leads. She was diagnosed with acute myocardial infarction. Although dopamine $(60 \mu \mathrm{g} / \mathrm{kg} / \mathrm{min})$ and norepinephrine $(0.8 \mu \mathrm{g} / \mathrm{kg} / \mathrm{min})$ were continuously administered, the patient's blood pressure did not respond to the medical treatment. Consequently, we decided to perform a peripheral veno-arterial ECMO (VA ECMO; EBS, Terumo, Tokyo, Japan) via left femoral artery and right femoral vein. Subsequently, she underwent emergency coronary angiography. A tight stenosis at the proximal and distal portion of right coronary artery (RCA) was shown. A guidewire was advanced into the RCA via a 6 F Brite Tip JR 4.0 guide catheter (Cordis, Miami Lakes, FL, USA). The lesion was predilated using a $2.5 \times 20$-mm Amadeus balloon (Eurocor, Bonn, Germany) at 10 atmospheres. Then, a $3.0 \times 30-\mathrm{mm}$ stent (Orsiro, Biotronik, Bülach, Switzerland) was placed successfully at 18 atmospheres in the distal portion of the RCA. Additionally, a $3.5 \times 26$-mm stent (Orsiro) was placed successfully at 18 atmospheres in the proximal portion of the RCA. Final angiographic findings were appropriate without showing any complications such as dissection, distal embolization, or slow flow. However, she was maintained on VA ECMO because of subsequent inadequate organ perfusion. The initial revolution per minute of the ECMO was adjusted to achieve at least one target, including a cardiac index greater than $2.2 \mathrm{~L} / \mathrm{min} /$ body surface area $\left(\mathrm{m}^{2}\right)$, central mixed venous saturation over $70 \%$, and mean arterial pressure (MAP) over $65 \mathrm{mmHg}$. Additionally, we used heparin for anticoagulation. Activated clotting time (ACT) was adjusted to 150-160 seconds (1.5 times normal, Hemochron Kaolin ACT Tests). After ECMO insertion,
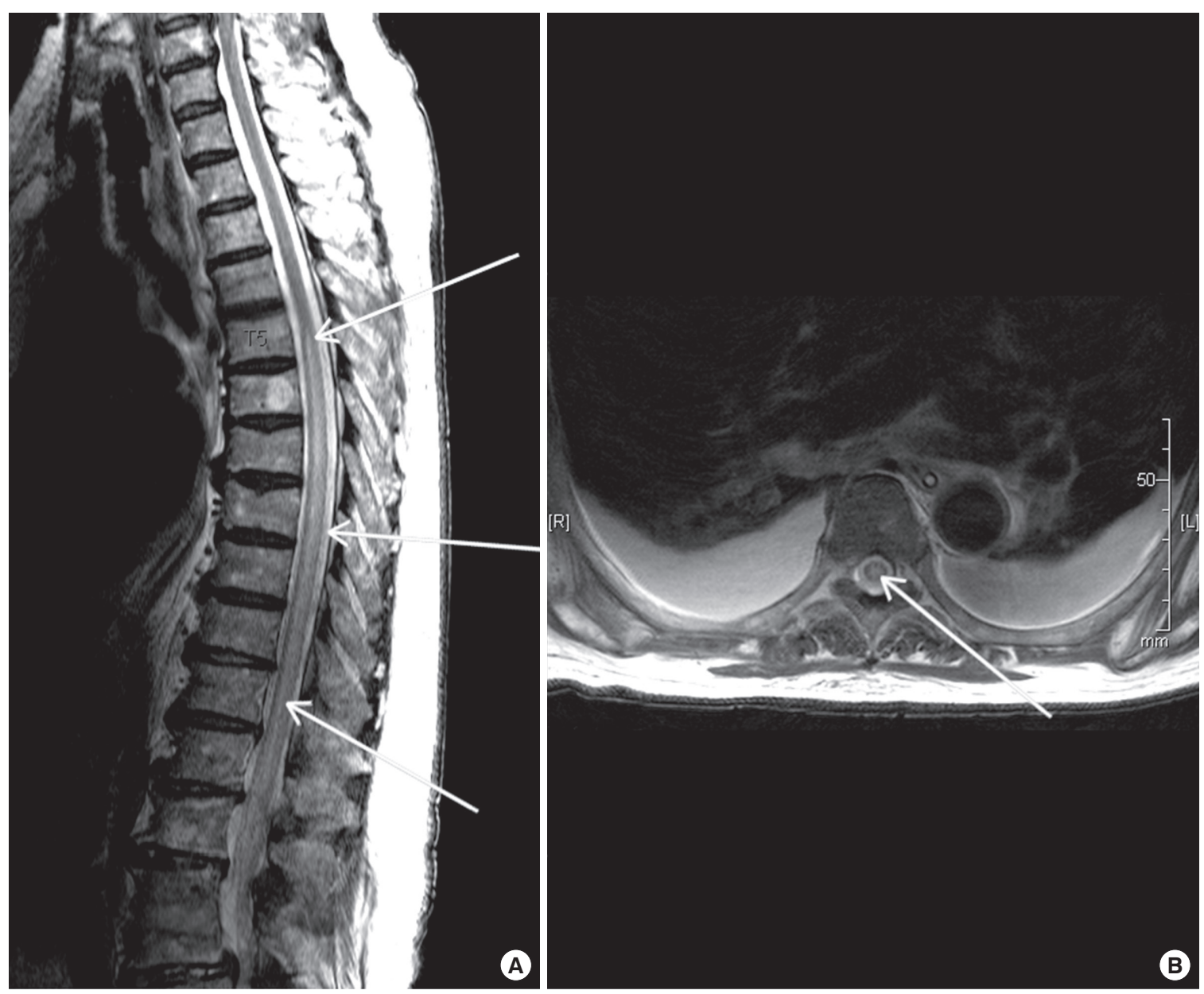

Figure 1. Axial (A) and sagittal (B) magnetic resonance imaging demonstrating diffuse swelling with increased T2 signal intensity (arrows) of the spinal cord (T5-conus medullaris). 
MAP was decreased to $50 \mathrm{mmHg}$. Fluid hydration (plasma solution $1 \mathrm{~L})$ and norepinephrine $(0.1 \mu \mathrm{g} / \mathrm{kg} / \mathrm{min})$ were started. The MAP was increased to $65 \mathrm{mmHg}$ within 1 hour. Vasopressor was discontinued. To prevent an accident for removal of the ECMO cannula (patient's Richmond Agitation-Sedation Scale [RASS] >2), we started sedative drug (remifentanil, 0.1 $\mathrm{mcg} / \mathrm{kg} / \mathrm{min}$ ). Distal weakness of the lower limbs with motor grade 0 defined as no movement was noted during a clinical examination on day 1 of VA ECMO. As the patient had to be sedated (RASS $\leq 2$ ), full neurologic assessment was difficult. Although the patient's neurologic symptom was persistent, her lower extremities were warm. Her pulse was palpable in the dorsalis pedis artery and the posterior tibial artery. We confirmed the patency of blood flow in the peripheral arteries of the lower extremities using color Doppler ultrasonography. Weakness of the lower limbs with motor grade 0 lasted 2 days with a sensory level of T6 on clinical examination after the removal of ECMO. Magnetic resonance imaging (MRI) was performed to exclude myelopathy. MRI of the spine demonstrated longitudinally extensive central T2-weighted signal abnormality extending from the level of $\mathrm{T} 5$ to the conus medullaris (Figure 1). The patient was medically stabilized; hence she underwent rehabilitation. However, there was no neurologic recovery.

\section{DISCUSSION}

Spinal infarction in patients undergoing ECMO is still an obscure and complicated issue. There are a few cases about the occurrence of spinal cord infarction related to circulatory support with a combination of VA ECMO with/without intra-aortic balloon pumping $[5,6]$. No neurologic sign or symptom is commonly found before VA ECMO insertion, suggesting a link between circulatory support period and neurological injuries. This is likely due to hypoperfusion of the spinal cord which is multifactorial in origin like: (1) low cardiac output states during the prolonged chest compression for cardiac arrest; (2) high vasopressor requirements causing vasospasm of the artery of Adamkiewicz and the consequential occlusion of retrograde oxygenated blood flow from VA ECMO; (3) thrombus generated in the extracorporeal circuit; and (4) vulnerable neurogenic blood volume and pressure mechanism due to various medications.

Spinal cord infarction can be divided into different clinical subtypes: anterior or posterior spinal artery infarctions due to mechanical stress, and central or transverse infarctions due to prolonged spinal-cord hypoperfusion [4]. Spinal MRI is the diagnostic tool of choice for spinal cord infarction [7]. Radiologic patterns for each subtype can be used to confirm the location and extension of the infarction. At central spinal cord infarction, MRI shows an ischemic lesion defined as a "pencillike" hyperintensity in sagittal T2-weighted images. MRI can also distinguish spinal cord infarction from other diseases, such as spinal cord compression, disc herniation, and hematoma. Currently, there is no treatment option except for supportive management for central spinal cord infarction. Since patients with spinal cord infarction have bare chance for recovery, early diagnosis and differential diagnosis are important [8]. In case of certain surgical patients, combination of hemodynamic augmentation and cerebrospinal fluid drainage has been evaluated for prevention of spinal cord infarction. If spinal cord infarction is suspected, MAP should be maintained above $90 \mathrm{mmHg}$ to raise spinal cord perfusion pressure [9]. We did not try such an attempt for our patient because of complications of volume overload and high dose of vasopressors that can impose heavy strain on the heart recovering from myocardial infarction. Although rehabilitation was initiated early in present case, the patient did not move without assistance after 4 weeks. Central spinal cord infarction in ECMO patient with cardiogenic shock requiring a vasopressor is an extraordinary complication associated with significant long-term morbidity. Several preventive strategies may help avoid this complication. First, adequate systemic blood pressure should be maintained before the patient's medical status is stabilized. Second, patients with VA ECMO must be carefully monitored with regular neurologic examinations of the lower limbs. If a patient does not require sedation, earlier detection is possible [5].

In conclusion, we report a case of spinal cord infarction in a patient with myocardial infarction complicated by cardiogenic shock during VA ECMO support. When lower limb weakness is found in ECMO patients with shock, spinal cord infarction should be considered as differential diagnosis.

\section{CONFLICT OF INTEREST}

No potential conflict of interest relevant to this article was reported.

\section{ORCID}

Beomsu Shin https://orcid.org/0000-0002-8449-0195 Jeong Hoon Yang ～https://orcid.org/0000-0001-8138-1367 


\section{REFERENCES}

1. Rigney L, Cappelen-Smith C, Sebire D, Beran RG, Cordato D. Nontraumatic spinal cord ischaemic syndrome. J Clin Neurosci 2015;22:1544-9.

2. Cheng MY, Lyu RK, Chang YJ, Chen RS, Huang CC, Wu T, et al. Spinal cord infarction in Chinese patients: clinical features, risk factors, imaging and prognosis. Cerebrovasc Dis 2008;26: 502-8.

3. Shamji MF, Maziak DE, Shamji FM, Ginsberg RJ, Pon R. Circulation of the spinal cord: an important consideration for thoracic surgeons. Ann Thorac Surg 2003;76:315-21.

4. Novy J, Carruzzo A, Maeder P, Bogousslavsky J. Spinal cord ischemia: clinical and imaging patterns, pathogenesis, and outcomes in 27 patients. Arch Neurol 2006;63:1113-20.

5. Samadi B, Nguyen D, Rudham S, Barnett Y. Spinal cord infarct during concomitant circulatory support with intra-aortic balloon pump and veno-arterial extracorporeal membrane oxygenation. Crit Care Med 2016;44:e101-5.

6. Oda T, Yasunaga H, Tsutsumi Y, Shojima T, Zaima Y, Nishino $\mathrm{H}$, et al. A child with influenza A (H1N1)-associated myocarditis rescued by extracorporeal membrane oxygenation. J Artif Organs 2010;13:232-4.

7. Weidauer S, Nichtweiss M, Lanfermann H, Zanella FE. Spinal cord infarction: MR imaging and clinical features in 16 cases. Neuroradiology 2002;44:851-7.

8. Robertson CE, Brown RD Jr, Wijdicks EF, Rabinstein AA. Recovery after spinal cord infarcts: long-term outcome in 115 patients. Neurology 2012;78:114-21.

9. Rubin MN, Rabinstein AA. Vascular diseases of the spinal cord. Neurol Clin 2013;31:153-81. 